\title{
AVALIAÇÃO DO EMPREGO DO GM1 APÓS LESÃO MEDULAR EXPERIMENTAL EM RATOS
}

\author{
EVALUATION OF THE USE OF GM1 AFTER EXPERIMENTAL SPINAL CORD INJURY IN RATS \\ EVALUANDO EL USO DE GM1 DESPUÉS DE LESIÓN EXPERIMENTAL DE MÉDULA ESPINAL \\ EN RATONES
}

Fabiano Inácio de Souza', Tarcísio Eloy Pessoa Bafros Filloo ${ }^{2}$, Alexandre Fogaça Cristante ${ }^{3}$

\begin{abstract}
RESUMO
Objetivo: Avaliar a eficácia do GM1, administrado por via intraperitoneal, após lesão medular experimental em ratos. Métodos: Foram utilizados 20 ratos da raça Wistar machos, adultos jovens, com média de idade de 20 semanas, pesando em torno de $350 \mathrm{~g}$, divididos em dois grupos de 10 animais. No grupo 1 foi administrado apenas soro fisiológico por via intraperitoneal. Ao grupo 2, administraram-se 30 mg/kg de GM1 diariamente por essa mesma via. As lesões foram produzidas seguindo-se o protocolo internacional MASCIS (Multicenter Animal Spinal Cord Injury Study), com o sistema NYImpactor. Os animais foram avaliados funcionalmente pela escala BBB nos dias 14, 28 e 42 após a lesão. O potencial evocado foi realizado em todos os animais, no $42^{\circ}$ dia após o trauma. Resultados: Utilizou-se um modelo de variância de dois fatores (ANOVA) e o teste $t$ de Student. As avaliações funcionais e por meio de potencial evocado não demonstraram diferença estatisticamente significante entre os dois grupos. Conclusão: O emprego de GM1 intraperitoneal não demonstrou resultados satisfatórios após lesão medular experimental.
\end{abstract}

Descritores: Traumatismos da medula espinal; Gangliosídeo G(M1); Ratos Wistar.

\begin{abstract}
Objective: To evaluate the efficacy of GM1, administered intraperitoneally after experimental spinal cord injury in rats. Methods: A total of 20 Wistar rats, young adults, males, with a mean age of 20 weeks, weighting about $350 \mathrm{~g}$ were divided into two groups of 10 animals. The group 1 was given only saline intraperitoneally. Group 2 received GM1 30mg/kg daily, also intraperitoneally. The lesions were produced by following the international protocol MASCIS (Multicenter Animal Spinal Cord Injury Study), using the system NY Impactor. The animals were evaluated functionally by the BBB scale at days 14, 28 and 42 after injury. The evoked potential was performed on all animals 42 days after trauma. Results: We used a two-factor variance model (ANOVA) and the Student $t$ test. The functional assessments as well as those by evoked potential showed no statistically significant difference between the two groups. Conclusion: The use of intraperitoneal GM1 was not efficient after experimental spinal cord injury.
\end{abstract}

Keywords: Spinal cord injuries; G(M1) Ganglioside; Rats, Wistar.

\section{RESUMEN}

Objetivo: Evaluar la eficacia del GM1, administrado por vía intraperitoneal, después de la lesión medular experimental en ratones. Métodos: se utilizaron 20 ratones de la raza Wistar, machos, adultos jóvenes, con una edad promedio de 20 semanas, peso de 350 gramos, aproximadamente, que se dividieron en dos grupos de 10 animales. En el grupo 1 se usó solamente suero fisiológico por vía intraperitoneal. En el grupo 2, se administró por vía intraperitoneal $30 \mathrm{mg} / \mathrm{kg}$ de GM1 por día. Las lesiones fueron producidas siguiéndose el protocolo internacional MASCIS (estudio multicéntrico de lesión de médula espinal en animales) por sistema NYImpactor. Los animales se evaluaron funcionalmente por la escala BBB en los días 14, 28 y 42 después de la lesión. El potencial evocado se realizó en todos los animales a los 42 días después del traumatismo. Resultados: se utilizó un modelo de variancia de dos factores (ANOVA) y la prueba $t$ de Student. Las evaluaciones funcionales y de los potenciales evocados no mostraron diferencias estadísticamente significativas entre los dos grupos. Conclusión: El uso de GM1 intraperitoneal no demostró resultados satisfactorios después de la lesión medular experimental.

Descriptores: Traumatismos de la médula espinal; Gangliósido G(M1); Ratones Wistar.

\section{INTRODUÇÃO}

A lesão medular grave encontra-se entre os grandes desafios da medicina, devido à inexistência de tratamento satisfatório.

Geralmente os indivíduos acometidos encontram-se na plenitude da idade produtiva, ocorrendo a transformação de uma vida ativa para um estágio de dependência de terceiros, diminuição da auto-estima, possibilidade de desenvolvimento de doenças, associados a elevados custos permanentes.
A incidência de trauma raquimedular nos EUA varia de 42,4 a 51,4 , por milhão de habitantes ao ano, com prevalência entre 220 e 285.000 casos $^{1,2}$. Elevados gastos são realizados nestes casos. As despesas com internação inicial aproximam-se de U\$282,245.00, enquanto as modificações da casa $(\bigcup \$ 21,000.00)$, do carro (de $\cup \$ 1,000$ a 65,000), cuidados diários (U\$21,000.00/ano) e trabalhistas (U\$57,000.00/ano) completam o montante de gastos permanentes ${ }^{3}$. Outras formas de lesão medular são as tumorais e as iatrogênicas.

1. Doutorando e Mestre em Ortopedia pelo Departamento de Ortopedia eTraumatologia da Faculdade de Medicina da Universidade de São Paulo

2. ProfessorTitular do Departamento de Ortopedia eTraumatologia da Faculdade de Medicina da Universidade de São Paulo - São Paulo, SP, Brasil.

3. Doutor e Médico Assistente do Grupo de Coluna do Instituto de Ortopedia eTraumatologia do HC/FMUSP - São Paulo, SP, Brasil.

Trabalho desenvolvido no Laboratório de Estudos do Traumatismo Raquimedular e Nervos do Instituto de Ortopedia eTraumatologia do HC/FMUSP.

Correspondência: Rua Dr. Ovídio Pires de Campos, 333, $8^{\circ}$ andar, Cerqueira César, CEP: 05403-010. São Paulo-SP. Brasil. Email: fabianoinacio@ usp.br

Recebido em 19/03/2011, aceito em 03/08/2011 
Há controvérsias sobre os benefícios da utilização de drogas após lesão medular, nas quais a metilprednisolona e o GM1 (monossialotetraesosilgangliosídeo) encontram-se em destaque.

A necessidade dos estudos experimentais visando a aplicabilidade clínica é notória e indispensável.

O objetivo deste estudo é avaliar a eficácia do GM1, administrado pela via intraperitoneal, após lesão medular experimental em ratos.

\section{MÉTODOS}

Foram utilizados 20 ratos da raça Wistar, machos, adultos jovens, com idade média de 20 semanas, peso em torno de 350g, provenientes do Centro de Bioterismo da Faculdade de Medicina da USP, divididos em dois grupos de 10 animais.

No grupo1 foi administrado diariamente $0,5 \mathrm{ml}$ de soro fisiológico pela via intraperitoneal, enquanto que no grupo 2 foi injetado, pela mesma via, 30mg/kg de $\mathrm{GM} 1^{4}$.

\section{Procedimento cirúrgico}

Os procedimentos cirúrgicos e o acondicionamento dos animais foram realizados no Laboratório de Estudos do Traumatismo Raquimedular e Nervos do Instituto de Ortopedia e Traumatologia da USP.

Os animais foram anestesiados com pentobarbital, na concentração de $60 \mathrm{mg} / \mathrm{kg}$, pela via intraperitoneal no terço inferior do abdômen. Em seguida, realizou-se a tricotomia dorsal, antissepsia com clorexidine, e incisão longitudinal na topografia de T7 a T12, dissecção por planos e exposição dos componentes posteriores da coluna dorso-lombar. As lâminas de T9 e T10 foram ressecadas, no sentido caudo-cranial.

\section{Lesão medular}

As lesões foram produzidas seguindo-se o protocolo internacional MASCIS (Multicenter Animal Spinal Cord Injury Study) ${ }^{5}$, pelo sistema NYImpactor ${ }^{6}$, com altura de 25 mm (Figuras 1 e 2). O sítio lesionado foi irrigado com soro fisiológico à temperatura ambiente, seguindo-se à inspeção, cauterização, aproximação dos tecidos e sutura da pele.

A efetividade da lesão era verificada através dos dados obtidos no gráfico formado (Figura 3), registrando-se individualmente e armazenando o padrão de cada imagem.

Administrou-se, subcutaneamente, $25 \mathrm{mg} / \mathrm{kg}$ de cefalotina ${ }^{6}$.

Os animais foram mantidos em gaiolas $(60 \times 40 \mathrm{~cm})$ em número de 3 ou 4, sendo fornecido ração e água ad libitum. As massagens para esvaziamento vesical pós-operatório foram efetuadas diariamente, assim como avaliação de possíveis complicações, como infecção urinária, autofagia e óbito.

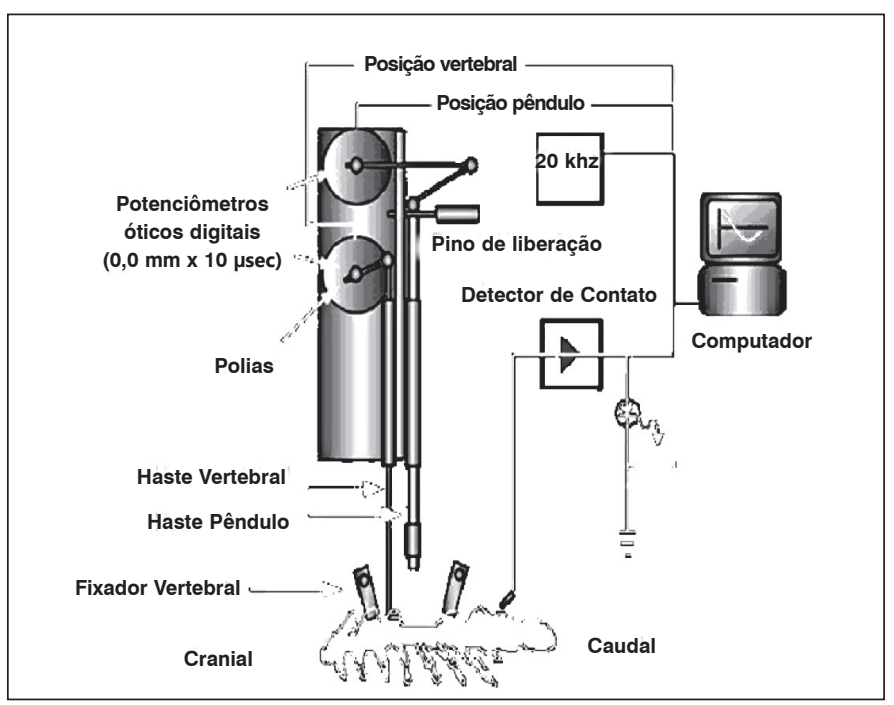

Figura 1. Diagrama do NYImpactor.

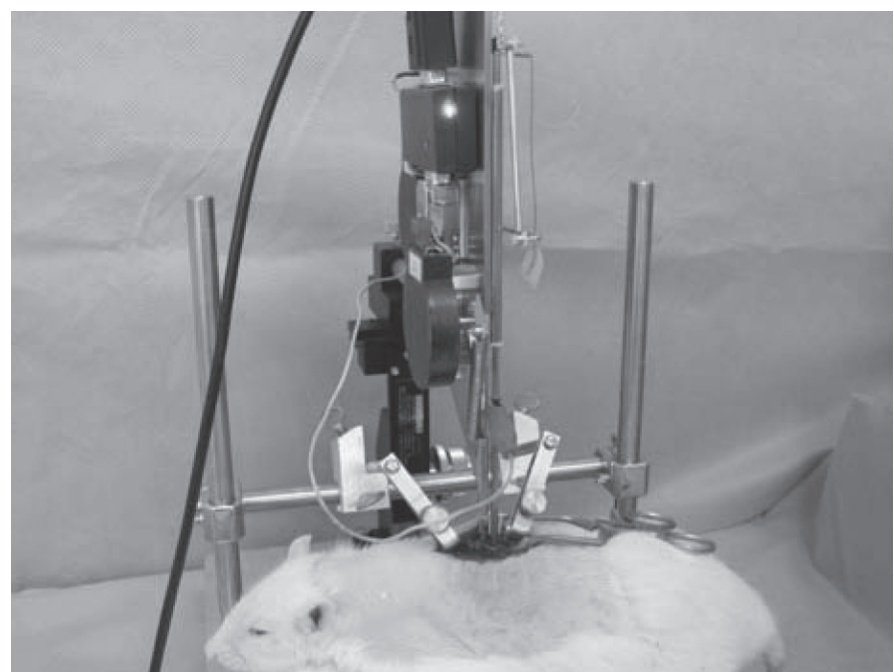

Figura 2. Momento da lesão medular.

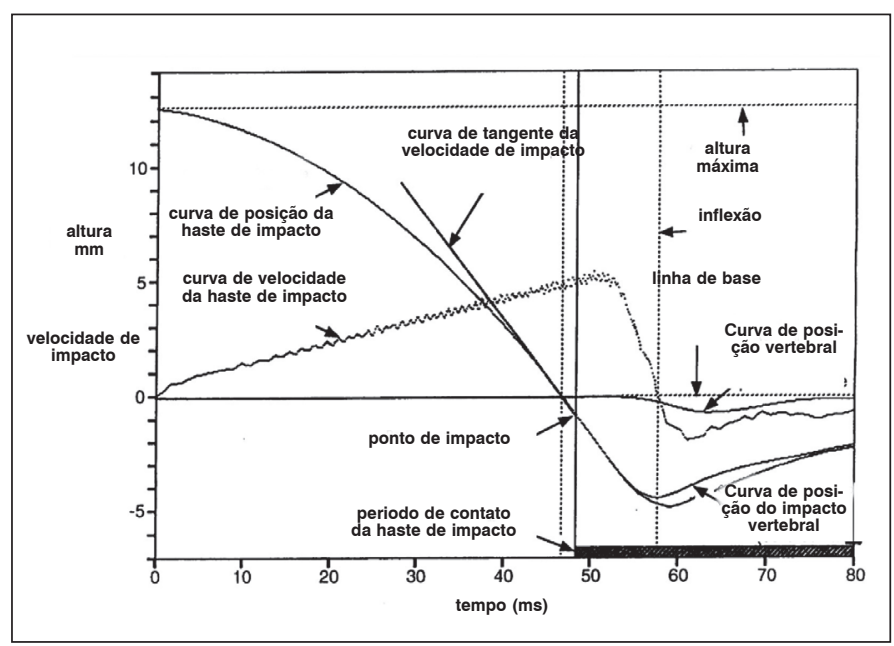

Figura 3. Gráfico padrão do NYImpactor.

\section{Avaliação funcional}

Os animais foram avaliados pela escala BBB (Basso, Beattie, Bresnahan $)^{7,8}$, nos dias 14, 28 e 42 após a lesão.

\section{Avaliação por potencial evocado motor}

O potencial evocado motor através da estimulação elétrica transcraniana ${ }^{9}$ foi realizado em todos os animais, 42 dias após 0 trauma, através de estímulo de único de 0,2 ms de duração, com intensidade de estímulo supramáxima. A captação das respostas musculares nas patas inferiores foi realizada por varredura com janela de $20 \mathrm{~ms}$, sensibilidade $2 \mathrm{mV} /$ div.

Em seguida, procedeu-se à eutanásia com administração intraperitoneal de pentobarbital $(140 \mathrm{mg} / \mathrm{Kg})$. Posteriormente foram disponibilizados seguindo os critérios do Colégio Brasileiro de Experimentação em Animais ${ }^{10}$.

\section{RESULTADOS}

Dois animais do grupo 1 e três do grupo 2 apresentaram autofagia do membro inferior ou óbito (um de cada grupo), sendo excluídos.

\section{Análise estatística}

Os valores médios dos escores BBB foram comparados utilizando um modelo de análise de variância (ANOVA) com dois fatores: grupo (1 e 2) e momento de avaliação (14, 28 e 42 dias), conside- 
rando medidas repetidas no fator momento de avaliação. A existência de efeito de interação entre estes fatores também foi avaliada.

A comparação entre as médias do potencial evocado motor (medidas das amplitudes) nos grupos 1 (Soro fisiológico) e 2 (GM1 intraperitoneal) foram comparadas por meio do teste $t$ de Student. $\mathrm{Na}$ análise da amplitude, as medidas analisadas correspondem às médias dos valores observados no membro inferior esquerdo (MIE) e direito (MID) de cada animal.

\section{Avaliação funcional}

A Tabela 1 e a Figura 4 apresentam medidas descritivas do escore BBB para cada grupo de estudo e momento de avaliação. De uma forma geral, observa-se um aumento do escore médio ao longo das avaliações, tanto para o grupo soro fisiológico quanto para o grupo GM1. No entanto, a $3^{a}$ avaliação mostra uma diferença maior entre os grupos, sendo a média (desvio padrão) igual a 14,8 $(4,2)$ no grupo soro fisiológico e 12,3 $(4,2)$ no grupo GM1. Apesar disso, a análise não indicou efeito significante de interação $(p=0,132)$. A existência de efeito de interação ocorre quando a diferença entre os grupos não é a mesma para os três momentos de avaliação ou, de forma equivalente, quando as diferenças entre os momentos de avaliação não são as mesmas para os diferentes grupos.

A análise também não mostrou diferenças estatisticamente significantes entre as médias dos grupos, ou seja, não houve efeito de grupo, com $p=0,637$.

Em relação ao momento de avaliação, a análise mostrou efeito significante $(p<0,001)$. Além disso, conclui-se que houve um aumento significante na média, tanto quando passamos da $1^{\mathrm{a}}$ avaliação $(2,0)$ para a $2^{\mathrm{a}}(7,5)$, como quando passamos da $2^{\mathrm{a}}$ para a $3^{a}$ avaliação $(13,6)$, ambas com $p<0,001$.

Tabela 1. Medidas descritivas para o escore BBB segundo grupos

\begin{tabular}{l|c|c|c|c|c|c}
\hline Grupo & Dia & Média & D.P. & Mediana & Mínimo & Máximo \\
\hline \multirow{3}{*}{$\begin{array}{c}\text { Soro fisiológico } \\
(\mathrm{N}=8)\end{array}$} & 14 & 1,8 & 1,7 & 2,0 & 0 & 4 \\
& 28 & 7,5 & 2,4 & 7,0 & 5 & 13 \\
& 42 & 14,8 & 4,2 & 15,0 & 7 & 20 \\
\hline \multirow{2}{*}{ GM1 } & 14 & 2,3 & 2,3 & 3,0 & 0 & 5 \\
$(\mathrm{~N}=7)$ & 28 & 7,4 & 3,3 & 7,0 & 3 & 13 \\
& 42 & 12,3 & 4,2 & 13,0 & 5 & 17 \\
\hline & 14 & 2,0 & 1,9 & 3,0 & 0 & 5 \\
Total & 28 & 7,5 & 2,8 & 7,0 & 3 & 13 \\
$(\mathrm{~N}=15)$ & 42 & 13,6 & 4,2 & 14,0 & 5 & 20 \\
\hline
\end{tabular}

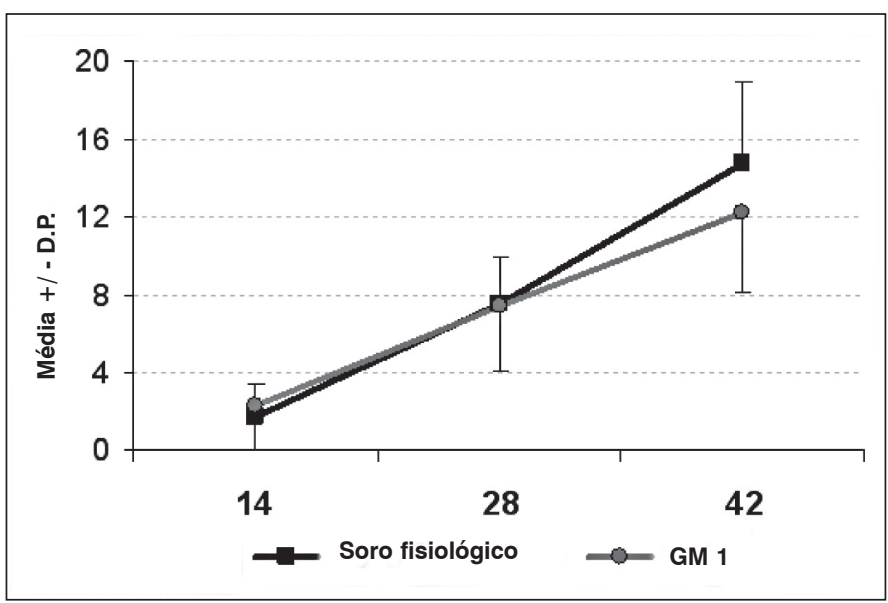

Figura 4. Médias e desvios padrões para os escores BBB, segundo grupo e dia de avaliação.

\section{Potencial evocado motor}

A Tabela 2 apresenta medidas descritivas da amplitude em cada grupo de estudo. No grupo 1, a amplitude teve média igual a 544,6 e variou de 81,0 a 1840,0 e, no grupo 2, foi de 392,9, variando de 120,0 a 1384,5 . Os dados apresentaram grande variabilidade, no entanto, há indicação de diferença significante entre as variâncias dos dois grupos (o teste de Levene para comparação entre as variâncias dos dois grupos forneceu $p=0,362$ ).

A análise não mostrou diferença estatisticamente significante entre as amplitudes médias dos grupos, com $p=0,590$.

Tabela 2. Medidas descritivas para a amplitude segundo grupos.

\begin{tabular}{|c|c|c|c|c|c|c|c|}
\hline & & $\mathbf{N}$ & Média & D.P. & Mediana & Mínimo & Máximo \\
\hline \multirow{3}{*}{$\begin{array}{c}\text { Grupo } \\
p=0,590\end{array}$} & Soro & 8 & 544,6 & 596,2 & 243,5 & 81,0 & 1840,0 \\
\hline & GM1 & 7 & 392,9 & 442,5 & 270,5 & 120,0 & 1384,5 \\
\hline & Total & 15 & 473,8 & 517,5 & 254,5 & 81,0 & 1840,0 \\
\hline
\end{tabular}

Valor de $p$ referente ao teste $t$ de Student.

\section{DISCUSSÃO}

As alterações decorrentes da lesão raquimedular são divididas em agudas e progressivas. As primeiras ocorrem imediatamente após o impacto traumático, onde o dano tecidual leva a morte celular local, incluindo neurônios, astrócitos, oligodendrócitos e células endoteliais, edema e isquemia. As alterações teciduais permanecem evoluindo, onde a ativação da cascata complementar ocasiona maciça migração de neutrófilos, levando à peroxidação lipídica e à formação de radicais livres, contribuindo para o dano neural.

Aumenta-se a concentração intracelular de cálcio e sódio e extracelular de potássio, determinando importante desequilíbrio físico-químico. A fase sub-aguda é marcada pela continuidade de morte celular, estimulada pela invasão de monócitos, macrófagos e linfócitos-T, e pela formação de tecido cicatricial pelos fibroblastos. A desmielinização distal acentua a morte dos oligodendrócitos ${ }^{11,12}$.

A taxa de mortalidade no primeiro ano após a fase aguda da lesão medular varia de $8 \%$ a $15 \%$. Estes indivíduos geralmente encontram-se em dependência ventilatória ou apresentam comorbidades pré-existentes, destacando-se doenças cárdio-pulmonares, endocrinológicas e o uso de drogas e álcool'13,14

$\mathrm{Na}$ fase crônica, os pacientes portadores de lesão medular estão propensos a desenvolver doenças psíquicas e físicas. 0 distúrbio psiquiátrico mais comum é a depressão, embora pode-se caracterizar o isolamento social, a perda da auto-estima e a sensação de inferioridade, como alterações comportamentais não necessariamente patológicas ${ }^{14}$.

Estes indivíduos apresentam alterações neurais e morfológicas no sistema gastrointestinal ${ }^{15}$, obesidade e suas comordidades, como hipertensão arterial sistêmica, cardiopatias, diabetes mellitus, úlceras de decúbio, vasculopatias ${ }^{16,17}$, contraturas músculo-tendíneas e disfunção sexual ${ }^{18}$. A dor crônica incide entre 11 e 94\% destes casos, aumentando sensivelmente a incidência de doenças psíquicas e dificuldade de terapias de manutenção ${ }^{19,20}$.

Desta forma, é imperativo o tratamento contínuo por equipes multidisciplinar e multiprofissional, buscando-se atenuar ao máximo o devastador impacto na qualidade de vida destas pessoas ${ }^{21,22}$.

Em casos com boa evolução, revisão da literatura demonstra baixo índice de retorno ao trabalho, com taxas variando de 11,5 a 74\%23.

Inúmeras linhas de pesquisa encontram-se em andamento em várias partes do mundo, onde um dos principais objetivos é a interferência na fase progressiva e degenerativa da lesão, visando-se impedir a evolução do quadro.

Algumas drogas são utilizadas clínica e experimentalmente, embora distante do consenso da comunidade científica. O GM1 é um glicolipídio presente nas membranas celulares de tecidos neurais, caracteriza-se pela capacidade de modulação e diferen- 
ciação celular às respostas adaptativas locais. É um neuroprotetor, antineurotóxico, indispensável na excitabilidade neuronal de fibras mielínicas e amielínicas, atua no desenvolvimento, crescimento, diferenciação e maturação neuronal, não requerem a presença de células gliais para sua atividade ${ }^{24-27}$, além de diminuir a intensidade da degeneração waleriana ${ }^{28}$.

Os testes funcionais, através do BBB, realizados com periodicidade de 14 dias demonstraram uma média superior no grupo 1 , embora sem efeito estatístico significante.

O potencial evocado motor por meio de estimulação elétrica transcraniana mensurando-se a amplitude, não demonstrou resultados estatisticamente significantes entre os grupos.

Marcon $\mathrm{RM}^{29}$, em 2010, testou o GM1 associado a oxigenoterapia hiperbárica, afirmando que houve efeito benéfico do emprego do gangliosídeo, embora, como neste estudo, não tenha se observado significância estatística.

Geisler et a. ${ }^{30}$ observaram que o GM1 utilizado nas primeiras 2 ho- ras e durante 18 a 32 dias, em pacientes portadores de lesão medular, sugere evidências de aumento da função neurológica após 1 ano.

Há necessidade de novos estudos experimentais empregando-se associações de fatores de crescimento, protetores neurais, dentre outras moléculas, na busca de melhores resultados no trauma medular.

\section{CONCLUSÃO}

As avaliações funcional e por potencial evocado não demonstraram resultados estatisticamente significantes do emprego do GM1 intraperitoneal na lesão medular pós-traumática experimental em ratos.

\section{AGRADECIMENTOS}

Nossos sinceros agradecimentos ao Dr. Ricardo Ferreira e ao Sr. Gustavo Bispo dos Santos, pelo indispensável auxílio. À empresa TRB Pharma, pela doação do GM1.

\section{REFERÊNCIAS}

1. Spinal cord injury facts and figures at a glance 2008. Disponivel em: http://www.spinalcord.uab.edu/show.asp?durki=116979. Acessado em 15 de junho de 2009.

2. Wyndaele $M$, Wyndaele JJ. Incidence, prevalence and epidemiology of spinal cord injury: what learns a worldwide literature survey? Spinal Cord. 2006;44(9):523-9.

3. Priebe MM, Chiodo AE, Scelza WM, Kirshblum SC, Wuermser LA, Ho CH. Spinal cord injury medicine. 6. Economic and societal issues in spinal cord injury.. Phys Med Rehabil. 2007:88(3):S84-8.

4. Tebet MA. Efeito da metilprednisolona e do gangliosídeo GM1 na lesão medular em ratos: análise funcional e histológica [tese]. São Paulo: Faculdade de Medicina da Universidade de São Paulo; 2002.

5. Dombourian MG, Turner NA, Gerovac TA, Vemuganti R, Miranpuri GS, Tureyen K, et al. B1 and TRPV-1 receptor genes and their relationship to hyperalgesia following spinal cord injury. Spine (Phila Pa 1976). 2006;31(24):2778-82.

6. Rodrigues NR. Padronização da lesão na medula espinal em ratos Wistar [tese]. São Paulo: Faculdade de Medicina, Universidade de São Paulo; 1999.

7. Basso DM, Beattie MS, Bresnahan JC. A sensitive and reliable locomotor rating scale for open field testing in rats. J Neurotrauma. 1995;12(1):1-21.

8. Barros Filho TEP, Molina AEIS. Analysis of the sensitivity and reproducibility of the basso, beattie, bresnahan (BBB) scale in wistar rats. Clinics. 2008;63(1):103-8.

9. Ferreira R, Oliveira AR, Barros FilhoTEP. Padronização da técnica para captação do potencial evocado motor em ratos através da estimulação elétrica transcraniana. Acta Ortop Bras. 2005;13(3):112-4.

10. Colégio Brasileiro de Experimentação Animal - COBEA. Legislação e ética, 2007. Dispo-

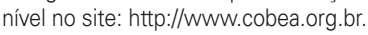

11. Trivedi A, Olivas AD, Noble-Haeusslein. Inflammation and spinal cord injury: infiltrating leukocytes as determinants of injury and repair processes. Clin Neurosc Res. 2006;6(5):283-92.

12. Sekhon LH, Fehlings MG. Epidemiology, demographics, and pathophysiology of acute spinal cord injury. Spine (Phila Pa 1976). 2001;26(24 Suppl):S2-12.

13. Krause JS, Carter RE, Pickelsimer EE, Wilson D. A prospective study of health and risk of mortality after spinal cord injury. Arch Phys Med Rehabil. 2008;89(8):1482-91.

14. Banerjea R, Sambamoorthi U, Weaver F, Maney M, Pogach LM, Findley T Risk of stroke, heart attack, and diabetes complications among veterans with spinal cord injury.

15. Arch Phys Med Rehabil. 2008;89(8):1448-53.

16. Kabatas S, Yu D, He XD, Thatte HS, Benedict D, Hepgul KT, et al. Neural and anatomical abnormalities of the gastrointestinal system resulting from contusion spinal cord injury. Neuroscience. 2008;154(4):1627-38.

17. Buchholz AC, Bugaresti JM. A review of body mass index and waist circumference as markers of obesity an coronary heart disease risk in persons with chronic spinal cord injury. Spinal Cord. 2005;43(9):513-8.

18. Maruyama Y, Mizuguchi M, Yaginuma T, Kusaka M, Yoshida H, Yokoyama K, et al. Serum leptin, abdominal obesity and the metabolic syndrome in individuals with chronic spinal cord injury. Spinal Cord. 2008;46(7):494-9.

19. Noonan VK, Kopec JA, Zhang H, Dvorak MF. Impact of associated conditions resulting from spinal cord injury on health status and quality of life in people with traumatic central cord syndrome. Arch Phys Med Rehabil. 2008;89(6):1074-82.

20. Raichle KA, Hanley M, Jensen MP, Cardenas DD. Cognitions, coping, and social environment predict adjustment to pain in spinal cord injury. J Pain. 2007:8(9):718-29.

21. Hanley MA, Raichle K, Jensen M, Cardenas DD. Pain catastrophizing and beliefs predict changes in pain interference and psychological functioning in persons with spinal cord injury. J Pain, 2008:9(9):863-71.

22. Curt $A$, Van Hedel HJA, Klaus D, Dietz V. Recovery from a spinal cord injury: significance of compensation, neural plasticity, and repair. J Neurotrauma. 2008;25(6):677-85.

23. Singh R, Dhankar SS, Rohilla R. Quality of live of people with spinal cord injury in Northern India. Int J Rehabil Res. 2008;31(3):247-51.

24. Lidal IB, Huynh TK, Biering-Sorensen F. Return to work following spinal cord injury: a review. Disabil Rehabil. 2007;29(17):1341-75.

25. Qiao GF, Cheng ZF, Huo R, Sui XH, LuYJ, Li BY. GM1 ganglioside contributes to retain the neuronal conductions and neuronal excitability in visceral and baroreceptor afferents. J Neurochem. 2008;106(4):1637-45.

26. Ledeen $\mathrm{R}$, Wu G. GM1 in the nuclear envelope regulates nuclear calcium through association with a nuclear sodium-calcium exchanger. J Neurochem. 2007;103(Suppl 1):126-34

27. Furian AF, Oliveira MS, Royes LFF, Fiorenza NG, Fighera MR, Myskiw JC, et al. GM1 ganglioside induces vasodilation and increaes catalase content in the brain. Free Radic Biol Med. 2007:43(6):924-32

28. Sokolova TV, Zakharova IO, Furaev VV, Rychkova MP, Avrova NF. Neuroprotective effect of ganglioside $\mathrm{GM} 1$ on the cytotoxic action of hydrogen peroxide and amyloid beta-peptide in PC12 cells. Neurochem Res. 2007;32(8):1302-13.

29. Oliveira ALR, Langone F. GM-1 ganglioside treatment reduces motoneuron death after ventral root avulsion in adults rats. Neurosci Lett. 2000 Oct 27;293(2):131-4 